\title{
Género y Cambio Climático
}

\section{Gender and Climate Change}

Luiza Simões Cozer

Universidade de Salamanca

luizacozer@gmail.com

\section{Resumo}

Actualmente los efectos del cambio climático son reconocidos en todo el mundo. Personas de diferentes capas sociales y de diversos países se manifiestan frente a este fenómeno porque ya están siendo afectados. El cambio climático tiene actualmente y aún tendrá mayores efectos e impactos diferenciados en los distintos grupos sociales, como género, edad, nivel económico y etnia. Por lo tanto, podemos afirmar que el cambio climático puede hacer que aumente la desigualdad en el mundo si no tenernos en cuenta sus orígenes y sus efectos. Así, es fundamental garantizar una mayor participación de toda la sociedad, pues se trata de un tema trascendental para las actuales y futuras generaciones.

Palabras-llaves: género, cambio climático, igualdad.

\section{Abstract}

Today the effects of climate change are recognized all over the world. People from different social classes and different countries care about this phenomenon because they are already being affected. Climate change has now and it will have major effects and different impacts on different social groups, like gender, age, economic status and ethnicity. Therefore, we can say that climate change can increase inequality in the world if we do not consider its origins and effects. Thus, it is essential to ensure greater participation of the entire society, because it is a transcedental issue for present and future generations.

Keywords: gender, climate change, equality. 


\section{Introducción}

Los efectos del cambio climático son reconocidos en todo el mundo, y son muy pocos los que no creen en la existencia de este fenómeno y de su aceleramiento, relacionado con el vínculo a las actividades antropogénicas.

Mujeres y hombres de diferentes capas sociales y de diversos países se manifiestan frente a este fenómeno porque ya están siendo afectados. La frecuencia e intensidad del fenómeno climático como consecuencia del cambio del clima está aumentando de manera considerable.

Se incrementa cada vez más la convicción de que los efectos del cambio climático son una amenaza seria para el desarrollo humano sostenible, pues tienen impacto en diversos sectores, como en la agricultura, la salud humana, la energía, la economía, la seguridad alimentaría, etc.

Es importante tener en cuenta que el cambio climático tiene actualmente y aún tendrá mayores efectos e impactos diferenciados en los distintos grupos sociales, como género, edad, nivel económico y etnia. Por lo tanto, podremos afirmar que el cambio climático puede hacer que aumente la desigualdad en el mundo si no tenernos en cuenta sus orígenes y sus efectos.

Frente a esta situación es fundamental garantizar una mayor participación de toda la sociedad, o sea, mujeres, hombres, jóvenes, niñas, niños, ancianas y ancianos, que debe ser la que conforma tanto los países desarrollados como los países en vías de desarrollo. Pues se trata de un tema trascendental para las actuales $\mathrm{y}$ futuras generaciones.

Para que podamos enfrentar y dar respuesta al problema del cambio climático de una manera real y eficiente, debemos considerar que es imprescindible la representación equilibrada en la toma de decisiones e intento de solución de problemas en todos los niveles ya sea local, regional, nacional o internacional.

El objetivo de este trabajo es enseñar los actuales avances conceptuales y metodológicos sobre las relaciones de género en el contexto del cambio climático, para proporcionar el entendimiento de la problemática socio-ambiental abordada desde una mirada más integral introducida por la perspectiva de género.

Señalamos, que el presente estudio es de fundamental relevancia una vez que aborda un tema tan actual como es la relación entre género y cambio climático. Así buscamos contestar a la siguiente pregunta: ¿Mujeres y hombres están afectados por el cambio climático de la misma manera?

\section{Consideraciones Sobre el Cambio Climático}

Según Domingues (p. 23) "el clima es consecuencia de las interacciones que se establecen entre los cinco componentes del sistema climático, la atmósfera, los océanos, las biosferas terrestre y marina, la criosfera (agua en estado sólido), y la superficie terrestre".

El clima de la Tierra nunca ha sido estático, puesto que con las alteraciones en el balance energético, el clima se somete a variaciones en todas las escalas temporales (desde decenios a miles y millones de años). Tales cambios son consecuencia de causas naturales, pero, en los últimos siglos, también es debido a la actividad de los seres humanos (DOMINGUES, p. 23).

Según Campos y Perez (2007) se llama cambio climático a la variación global del clima de la Tierra a lo largo del tiempo. La Convención Marco de Naciones Unidas sobre Cambio Climático lo define como:

un cambio de clima atribuido directa o indirectamente a la actividad humana, que altera la composición de la atmósfera mundial y que se suma a la variabilidad natural del clima observada durante períodos de tiempo comparables.

De acuerdo con el Grupo Intergubernamental de Expertos sobre el Cambio Climático 2007 (IPCC Intergovernmental Panel on Climate Change p. 2), el cambio climático se refiere a "cualquier cambio en el clima, ya sea por su variabilidad natural o como resultado de la actividad humana".

De modo natural, a lo largo de la historia del planeta, el clima ha cambiado. Los cambios naturales no significan obligatoriamente que hayan sido suaves, lineales e inocuos para las especies vivas que lo han habitado, podemos citar como ejemplo el caso de la desaparición de los dinosaurios que pudo ser resultado de un riguroso cambio en el clima, teniendo como causa la colisión de un gran meteorito con la tierra (CAMPOS y PEREZ, 2007).

De acuerdo con Hardy (2005), cambio climático es el cambio del clima imputado directamente o indirectamente a actividades humanas que cambien la composición de la atmósfera, y desarrollen una adición a la variación natural del clima. Lo que está sucediendo es justamente esto, un cambio de la composición de los gases en la atmósfera a través del aumento de la emisión de Gases de Efecto Invernadero 
producidos por actividades antropicas, aumentando la temperatura del Planeta.

Según Campos y Pérez (2007, p. 4):

las medidas más recientes informan que la Tierra está absorbiendo más energía de la que emite al espacio, se cree que esto sucede por el aumento de gases de efecto invernadero en la atmósfera, siendo la causa del calentamiento global del Planeta.

Los glaciares y la cubierta de nieve están disminuyendo en los hemisferios; la temperatura media del aire ha tenido una tendencia lineal de aumento positivo de $0,74^{\circ} \mathrm{C}$ de $1906-2005$. Desde 1961, a través de observaciones de científicos se ha constatado que la temperatura media de los océanos ha aumentado hasta profundidades mayores a los $3000 \mathrm{~m}$ de profundidad, y se piensa que ha absorbido el $80 \%$ del calor añadido (el calentamiento está provocando su expansión térmica y contribuyendo al aumento del nivel del mar); el nivel medio global del mar aumentó aproximadamente $1.8 \mathrm{~mm}$ por año entre 1961 a 2003; pérdidas de hielo desde Groenlandia y Antártica han sido, probablemente, un aporte para el aumento del nivel del mar entre 1961 y 2003 (BALDASANO, 2007).

Según Velayos (2008), no hay dudas de que se han verificado ya varios cambios en el clima, tales como primaveras más tempranas y duraderas; cambios en el hielo y en la temperatura del Ártico; en la cantidad de precipitaciones; en la cantidad de sal de los océanos; en los patrones de viento y en algunos aspectos de tiempo extremo, que abarcan el aumento de las sequías y de precipitaciones fuertes, y también las olas de calor y de la intensidad y el número de veces que suceden los ciclones tropicales.

El cambio climático desarrollará consecuencias significativas en el Planeta; pero sucede que la proporción, la velocidad y cuales sean sus consecuencias, aún provocan dudas al respecto. Esta es una situación a la que la sociedad postindustrial no está acostumbrada, pues una característica clave de esta sociedad es justamente el control del futuro, la prevención de los riesgos.

La opinión general de las investigaciones sobre el calentamiento del clima, es que se desarrollará una serie de problemas ambientales en el futuro, como indica el Cuarto Informe del IPCC: elevación en los próximos cien años de entre $1,8^{\circ} \mathrm{C}$ y $4^{\circ} \mathrm{C}$ de la temperatura media de la Tierra; aumento de precipitaciones catastróficas; fusión de casquetes polares, y aunque ocurra sólo de manera parcial, ocasionará el aumento del nivel de las aguas del mar y de los océanos; inundaciones en regiones como sudeste de Asia, de Pakistán hasta Vietnam, con riesgos de desaparición de estados insulares del Caribe, el Océano Indico y el Pacífico. Destacamos que las doscientas islas del delta de los Sundarbans, entre India y Bangladesh están amenazadas; mayor escasez de agua, principalmente en el sur de Europa y en África; consecuencias para la salud humana teniendo algunas causas como olas de calor, aumento de la malnutrición y de las enfermedades asociadas a ésta, tormentas e inundaciones con efectos en el agua dulce por la contaminación y aumento de diarreas, propagación de enfermedades como malaria, aumento del asma, mayor concentración de los niveles de ozono, generando afecciones cardiorrespiratorias; cambios en los ecosistemas, generando desaparición de especies animales y vegetales; cambios en la productividad agraria y pesquera; flujos migratorios de población; aumento de fenómenos como ciclones, huracanes, etc.

El principio de la precaución tendrá que funcionar para frenar la utilización de los combustibles fósiles, y promover la sustitución de estos por energías renovables, pues el uso de los combustibles fósiles llevan a un estado de incertidumbre en cuanto a los efectos de la aceleración del cambio climático.

El Principio de la Precaución es el principio donde se establecen medidas antes incluso de que se tenga una certeza científica al respecto de posibles daños, o sea, es el principio in dubio pro ambiente. Los riesgos totalmente o parcialmente desconocidos, como es el caso del cambio climático llevan a la incertidumbre, así como medida de precaución se debe tener en cuenta, qué riesgos la sociedad puede o está dispuesta a soportar.

\section{Crisis Climática y Vulnerabilidad}

En relación a la crisis climática y la vulnerabilidad, es importante decir que las comunidades más vulnerables son las más pobres, principalmente las ubicadas en zonas de alto riesgo, que dependen de recursos como agua y alimento local y estos son afectados por el cambio climático. Tal hecho hace con que tengan menos recursos para adaptarse a los cambios (VELAYOS, 2008).

Así, situaciones de, por ejemplo, sequías, inundaciones y tormentas suelen ser experiencias muy duras para las personas afectadas, puesto que ponen en riesgo sus vidas y les causa gran inseguridad. Todavía las crisis climáticas erosionan las oportunidades a largo plazo para el desarrollo humano al debilitar la productividad y destruir poco a poco las capacidades humanas (AGUILAR, CASTAÑEDA y CHAO, 
2008,). Según Rubio (2006), es importante señalar que es una tarea necesaria para quien busca afrontar el cambio climático relacionar la lucha contra el cambio climático a las políticas de erradicación de la pobreza, de transferencia e inversión de tecnología, de economía, de comercio internacional o de seguridad.

Un ejemplo de la vulnerabilidad/fragilidad humana ante el cambio climático, además en un país desarrollado, es el huracán Katrina. Es importante citar, que en todos los países desarrollados “está aumentando la inquietud pública respecto de la exposición a riesgos climáticos extremos y esa inquietud aumenta con cada inundación, tormenta y ola de calor". Sin embargo, los desastres suceden mucho más en los países pobres. Entre los años 2000 y 2004, por ejemplo, más o menos 262 millones de personas fueron afectadas por desastres climáticos todos los años y más del 98\% de ellas vivían en países en desarrollo (AGUILAR, CASTAÑEDA y CHAO, 2008, p.31).

Destacamos que los más vulnerables al cambio climático son las niñas y los niños y las mujeres. En relación a los primeros, de acuerdo con Gordon, Mackay y Rehfuess (2004) es muy elevado el número de niñas y niños entre cero y cuatro años que murieron en 2002 como consecuencia de causas relacionadas con el clima. La salud de niñas y niños, en opinión de Velayos (2008, p. 28): "sufrirá como resultado de las olas de calor, el incremento de la contaminación y las inundaciones, además de incremento de afecciones como la malaria".

En relación a las mujeres, ellas se ven afectadas de diferentes modos por el cambio climático y por los eventos climáticos extremos que muchas veces se traducen en desastres, en virtud de sus roles sociales, de la discriminación y de la pobreza (AGUILAR, CASTAÑEDA y CHAO, 2008).

Es importante mencionar que, según Velayos (2008, p. 29), "para tener éxito en las políticas ante el cambio climático, habría que tener mucho más en cuenta a las mujeres, cosa que no ocurre por el momento".

Además, las mujeres deben ser incluidas, porque tienen diferentes perspectivas y experiencias con las que pueden contribuir (como implementar medidas de adaptación) y no porque sean 'más vulnerables' (AGUILAR, CASTAÑEDA y CHAO, 2008).

De acuerdo con Duddy $\left(2010, \mathrm{~s} / \mathrm{p}^{1}\right)$ es de gran relevancia considerar el género cuando se analizan los asuntos del cambio climático:

El cambio climático no es un proceso neutral; en primer lugar, las mujeres en general son más vulnerables a los efectos del cambio climático, siendo una razón de esto que ellas conforman la mayoría de las personas pobres del mundo y dependen más que proporcionalmente de los recursos naturales que son amenazados. Los cambios tecnológicos y los instrumentos que están siendo propuestos para mitigar las emisiones de carbono, y que se presentan como neutrales al género, de hecho tienen un sesgo de género y podrían afectar negativamente a las mujeres o pasarlas por alto.

En relación a las condiciones climáticas extremas, en situaciones de tormentas, inundaciones y ciclones, son las mujeres quiénes mantienen unida a la familia, tienen la responsabilidad de luchar contra la devastación y la destrucción. En situaciones de catástrofes, la tarea de nutrir a la familia, principalmente a niñas y niños pequeños y también proveer los elementos cotidianos esenciales, también es a menudo sobrellevada en gran medida por las mujeres.

Además, es importante destacar que el enfoque de género en los efectos de desastre no aparece destacado. Cuando suceden los desastres, dañan a comunidades enteras, pero, en la mayoría de los casos son las mujeres las más afectadas. Con regularidad las inundaciones tienen más víctimas femeninas, una vez que su movilidad es limitada y no se les ha enseñado a nadar ${ }^{2}$. Por ejemplo, cuando en 1991 en Bangladesh sucedió un ciclón y una inundación devastadores, la tasa de mortalidad fue cinco veces superior entre las mujeres (UNDP, 2008).

En relación a los desastres, es importante mencionar que después de un desastre, "el acceso al crédito necesario para la reconstrucción se puede ver limitado por las restricciones que tienen las mujeres respecto de derechos legales a la tierra y a la propiedad" (AGUILAR, CASTAÑEDA y CHAO, 2008, p. 23).

Por lo tanto, la destrucción de la capa de ozono percibida en los 70 del siglo pasado y los efectos del 'smog' ${ }^{\prime 3}$ fueron el primer señal a la comunidad mundial de que la emisión ilimitada de algunos gases podrían traen efectos irreversibles a todas a las especies humanas y no humanas. A partir de eso es fácil percibir que la emisión desenfrenada de los gases de efecto invernadero están cambiando la temperatura media de la Tierra a un ritmo acelerado y que estas consecuencias pueden ser más desastrosas que la de los Clorofluorcarbonetos (CFCs) o del 'smog'.

La verdad es que la emisión desenfrenada de los gases de efecto invernadero provoca el llamado 
cambio climático provocado y con él una serie de consecuencias desastrosas en todos los puntos del Planeta. Este cambio climático, como todos los procesos que ocurrieron en la historia de la Tierra, presupone una ocurrencia desigual de los desastres, esto informado por los expertos sobre el tema.

Los países que más emitieron gases de efecto invernadero fueron los países 'desarrollados'. Las malas consecuencias del cambio climático serán percibidas en mayor número en los países en vías de desarrollo. Muchos motivos llevan a esto: o carencia de estos países en soportar fenómenos de la naturaleza o porque los efectos de acuerdo con los científicos ocurrirán más en el hemisferio sur. Pero también debemos considerar que muchas veces el cambio del clima es generado por los países ricos, pues son ellos que más explotan el medio ambiente y también no respetan los países en vías de desarrollo.

El escenario de la pobreza de los países del hemisferio sur enseña como protagonistas las mujeres que están más expuestas a los efectos del cambio del clima, ya que debido a un histórico cultural de opresión por parte de la sociedad machista cabe a las mujeres el cuidado de las niñas y los niños, del hogar, de las ancianas y los ancianos, en muchos sitios de la agricultura, reciben una educación diferente en casa, etc. Además, las mujeres están más expuestas a las enfermedades y a las malas condiciones de salud pública.

\section{El Concepto de Género}

En cuanto al concepto de género ${ }^{4}$, nos gustaría, primeramente, aclarar que lo que pretendemos en este apartado no es entrar en el inmenso debate (tanto lingüístico como teórico) que hay sobre el término. Buscamos solamente enseñar un concepto general, que desde nuestro entendimiento es lo que importa demostrar en este trabajo, para comprender las desigualdades existentes entre mujeres y hombres y las consecuencias que generan en relación al cambio climático.

Según Benería apud Murillo (1996, p. 14), se puede conceptuar género como "un conjunto de creencias, rasgos personales, actitudes, sentimientos, valores, conductas y actividades que diferencian al hombre de la mujer a través de un proceso de construcción social".

Scott (1995) rechaza claramente explicaciones biológicas, como aquellas que encuentran un denominador común, para diversas formas de subordinación femenina, en los hechos de que las mujeres tienen la capacidad para la reproducción y de que los hombres tienen una fuerza muscular superior.
Género es una forma de referirse a las 'construcciones culturales', a la creación totalmente social de ideas sobre los papeles adecuados a los hombres y a las mujeres, o sobre sus identidades subjetivas.

Por lo tanto, podemos decir el género trata de una categoría de análisis que destaca la construcción cultural de la diferencia sexual, que permite diferenciar aquellas características fisiológicas de aquellas características sociales, psíquicas e históricas que culturalmente se atribuye a la feminilidad y a la masculinidad. Por lo tanto, se asocia género a los papeles sociales atribuidos a mujeres y hombres, sin tener en cuenta características biológicas.

Además, debemos decir que en todas las sociedades existe una construcción cultural de lo femenino y de lo masculino, de lo que cada persona puede y debe ser de acuerdo con su sexo. Y cada sociedad tiene un conjunto de normas, tradiciones y valores que convierte en más o menos flexible esta atribución de lo femenino a las mujeres y de lo masculino a los hombres, creando sistemas de control y represión para quien pasa de los límites permitidos en los comportamientos de género.

En este trabajo es muy importante destacar, también, desde un análisis antropológico de la cultura, que:

Todas las culturas elaboran cosmovisiones sobre los géneros $\mathrm{y}$, en ese sentido, cada sociedad, cada pueblo, cada grupo y todas las personas, tienen una particular concepción de género, basada en la de su propia cultura. Su fuerza radica en que es parte de una visión del mundo, de su historia $y$ sus tradiciones nacionales, populares, comunitarias, generacionales y familiares. Forma parte de concepciones sobre la nación y del nacionalismo; cada etnia tiene su particular cosmovisión de género y la incorpora además a la identidad cultural y a la etnicidad, de la misma manera que sucede en otras configuraciones culturales. Es importante identificar las diversas cosmovisiones de género que coexisten en cada sociedad, cada comunidad y cada persona. Es posible que una persona a lo largo de su vida modifique su cosmovisión de género simplemente al vivir, porque cambia la persona, porque cambia la sociedad y con ella pueden transformarse valores, normas y maneras de juzgar los hechos (LAGARDE, 1996, p. 14). 
La citación previa es muy importante para este trabajo, pues debemos tener en cuenta que las diferencias culturales influencian mucho en cada sociedad y eso tiene muchos resultados, pues en las sociedades machistas y cerradas las mujeres van a tener menos espacio y poder, más desigualdad. Por eso, en el estudio sobre género y cambio climático debemos considerar las diferentes visiones en relación al género, pues eso va a determinar diferentes roles y papeles.

\section{Igualdad de Género}

Al tratar sobre mujeres y cambio climático, es imprescindible hablar de igualdad. Así, mencionamos que las mujeres buscan, a través de la igualdad, además de obtener sus derechos, dirimir la discriminación que sufren y también tener las mismas oportunidades que los hombres, para poder tener mayor acceso a liderazgo, poder y desarrollo.

Además, la Declaración del Milenio de las Naciones Unidas, entre los Objetivos de desarrollo del Milenio $\left(\mathrm{ODM}^{5}\right)$, en el objetivo III.20 destaca que es necesario "promover la igualdad entre los sexos y la autonomía de la mujer como medios eficaces de combatir la pobreza, el hambre y las enfermedades y de estimular un desarrollo verdaderamente sostenible".

Así, es importante mencionar el concepto de igualdad de género, que de acuerdo con el proyecto europeo de investigación 'Multiple Meanings of gender equality'6, desarrollado en la Universidad Complutense de Madrid, igualdad de género es

Situación en la que mujeres y hombres tienen iguales derechos y oportunidades de jure $\mathrm{y}$ de facto y participan por igual en todas las esferas de la vida pública y privada, libres para desarrollar sus capacidades y de tomar decisiones.

Así, lo que se busca a través de la igualdad de género es que los distintos comportamientos, objetivos y necesidades de las mujeres y de los hombres sean considerados, valorados y probados de igual forma. Esto no pretende sugerir que hombres y mujeres deben ser transformados en iguales, sino que sus derechos, oportunidades, responsabilidades no dependan de su género.

\section{Desigualdades de Género}

De la misma manera, a partir de los años ochenta, que se ha buscado el género en la literatura, en la historia, en el arte o en la ciencia, también se han examinado, según García apud González (2008, p. 115), las “desigualdades socioespaciales y ambientales derivadas de los diferentes roles sociales asignados a hombres y mujeres".

Pero, infelizmente, la "división sexual de las sociedades humanas se hace especialmente visible en la desigual distribución de hombres y mujeres en los espacios públicos y los espacios privados" (GARCÍA apud GONZÁLEZ, 2008, p. 115).

Por eso cabe decir que los hombres poseen una fuerte y establecida relación con el espacio público y con el mercado de trabajo, lo que les ofrece ventajas sociales, económicas y políticas a las que las mujeres tienen mucha más dificultad para acceder. El patriarcado radicado en las relaciones sociales, que transciende el espacio privado de la familia y alcanza el espacio público, incentiva aún más la feminización de la pobreza.

Además, el acceso diferencial de las mujeres y de los hombres a los bienes o a los recursos sociales y físicos es una de las dimensiones claves de la desigualdad de género. La posición social de las mujeres en muchas situaciones significa que los papeles que se esperan de ellas son frecuentemente de cuidado ${ }^{7}$ y reproducción, centrado en torno a la casa y la comunidad local, y no en la esfera pública. Esto no significa que las mujeres no poseen papeles cruciales en la producción agrícola $u$ otras actividades esenciales a los medios de subsistencia sostenible y economía nacionales. Pero, los papeles que les son atribuidos son generalmente menos visibles y atraen menos reconocimiento público que el trabajo de los hombres (BRODY, DEMETRIADES y ESPLEN, 2008).

Señalamos que para las mujeres es transmitida la noción de productividad apenas en el ámbito doméstico, no mencionándose sus capacidades a fin de que ellas puedan participar en el ámbito público. Así, el prejuicio de género se inicia desde muy temprano, pues chicas y chicos son inducidos a hacer o dejar de hacer algunas actividades en virtud de una concepción culturalmente aconsejada 'de lo que es' papel masculino y 'de lo que es' papel femenino.

Se espera que las mujeres - particularmente aquellas en posición de pobreza y las rurales - asuman la responsabilidad primaria para la subsistencia de sus familias. Pero, frecuentemente no reciben un salario, y son excluidas frecuentemente de las decisiones sobre los gastos de la casa o sobre la educación de hijas e hijos. La expectativa de que las niñas ayudarán a sus madres en las tareas de la casa y a cuidar de los hermanos pequeños, significa que son más probables de ser excluidas de las oportunidades de obtener una 
educación que los chicos. Las mujeres que consiguen un salario ganan a menudo menos que los hombres, dejándolas más vulnerables a los cambios en su entorno de trabajo causado por fenómenos externos, incluyendo el cambio del clima.

En relación a la desigualdad de género y cambio climático, es importante señalar que también existe la necesidad de tener en cuenta la desigualdad de género frente al cambio climático en países con mayor nivel de desarrollo, pues aunque tengan una realidad con una sociedad más igualitaria en diversos sentidos, las desigualdades de género aún existen.

Las desigualdades sociales tienen graves consecuencias en la vida de muchas mujeres, pues "limitan su acceso a la propiedad de la tierra, la vivienda, la educación, la salud y la participación en la esfera política y de toma de decisiones, es decir, limitan sus libertades humanas y reducen sus opciones" (AGUILAR, CASTAÑEDA y CHAO, 2008, p. 20).

Según PERAL apud ENCISO (2007, s/p p $^{8}$ “el cambio climático, que ya es irreversible, representa una amenaza importante que ampliará las desigualdades sociales y revertirá el desarrollo humano."

Según el IPCC 9 (apud Women for Climate Justice 2010), "los impactos del cambio climático se distribuirán de manera diferenciada entre las regiones, generaciones, edades, clases, ingresos, ocupaciones y sexos".

Hay que tener en cuenta algunos factores en la relación diferenciada de mujeres y hombres, tales como el nivel de dependencia de los recursos ambientales para la subsistencia; las relaciones desiguales en el uso, acceso y control de recursos, y en la distribución de beneficios; la propiedad, protección $\mathrm{y}$ derechos sobre los recursos; y conocimientos diferenciados sobre los recursos, productos derivados y problemas ambientales.

Teniendo en cuenta las desigualdades de género en el contexto del cambio climático, es importante la inclusión de las mujeres en la sociedad, pero en virtud de la exclusión social de las mujeres, debemos preocuparnos por su inserción.

\section{Género y Cambio Climático: Relacionando los Vínculos Existentes}

En este apartado vamos a estudiar la relación entre género y cambio climático. Para eso, pensamos que es interesante analizar dicha relación basada en los ocho Objetivos de desarrollo del Milenio (ODM) de la ONU, para así desarrollar este tema y mostrar que los efectos del cambio climático tienen implicaciones diferenciadas para mujeres y hombres, y cómo éstas influyen en el logro de los ODM.

En principio, podemos afirmar que la degradación del medio ambiente afecta a todas las personas, "pero no afecta de igual forma a varones y a mujeres. El deterioro del hábitat produce movilidad, desplazamientos y cambios en la estructura familiar". Así, constatamos que mujeres y niñas, sea en áreas urbanas o rurales, enfrentan una mayor carga de trabajo y prevalece su vulnerabilidad (CAMPOS y PEREZ, 2007, p. 19).

Tal situación se encuentra relacionada y puede ser justificada por los roles atribuidos a las mujeres, como puede ser la relación a la esfera privada, y además están culturalmente vinculadas al ámbito doméstico que incluye el cuidado de la familia, y también son características normalmente atribuidas a las mujeres la debilidad, la incapacidad, etc.

Campos y Perez (2007, p. 19-20) aducen que las mujeres dedican:

Mayores horas en la recolección de forraje, combustible y acarreo de agua para el cultivo o consumo familiar; están más expuestas a la contaminación por combustión y contaminación difusa; sufren mayor inseguridad y están expuestas a múltiples formas de violencia; de niñas dejan los estudios para ayudar en el hogar y en la siembra; no son propietarias de las tierras que cultivan y no pueden acceder a créditos ni a información que les permita producir mejor. Las barreras a las que se enfrenta son tanto la fuerte tradición patriarcal como el sistema legal que no otorga los mismos derechos a hombres y mujeres y que no instaura mecanismos para establecer tanto la igualdad formal como la igualdad real.

Así, constatamos que las desigualdades sociales entre mujeres y hombres afectan a las mujeres de una forma diferente, pues siempre son las más perjudicadas, una vez que son ellas las que tienen el acceso a la educación, a la salud, igualdad de oportunidades, participación en la sociedad, etc., limitado. En relación al cambio climático, no podría ser diferente, y uno de los factores que perjudica seriamente a las mujeres es la situación de pobreza en que viven y que son la gran mayoría.

El primer $\mathrm{ODM}^{10}$ es 'erradicar la pobreza extrema y el hambre'. Es importante mencionar, de una manera general, el concepto de pobreza, que es muy complejo. 
Para definir el concepto de pobreza, es necesario comprender que la pobreza puede ser entendida en varios sentidos, pues sobre ello no hay consenso; lo que hace que sea difícil presentar una definición correcta y única.

Un estudio hecho por Narayand (2000, p.6), donde se analizó dicha situación en muchos países, define la pobreza a través de la opinión de personas pobres de varias partes del mundo, como:

Pobreza é fome, é falta de abrigo. Pobreza é estar doente e não poder ir ao médico. Pobreza é não poder ir à escola e não saber ler. Pobreza é não ter emprego, é temer o futuro, é viver um dia de cada vez. Pobreza é perder o seu filho para uma doença trazida pela água não tratada. Pobreza é falta de poder, falta de representação e liberdade.

Por ello, constatamos que la pobreza se traduce en hambre, malnutrición, malas condiciones de salud, tasas de morbilidad y mortalidad elevadas, instrucción deficiente, hábitat precario e insano y desigualdad.

Es importante señalar que si analizarnos atentamente los dos párrafos anteriores, podremos perfectamente hacer una comparación entre la definición de pobreza y de desigualdad de género, pues ha sido mencionada además de la falta de lo que es necesario para las necesidades básicas, la incapacidad para participar en la sociedad, la falta de 'voz' y de poder. Eso no es nada novedoso para este estudio pues en el capitulo anterior hablamos de desigualdad de género $\mathrm{y}$ verificamos que las mujeres están muy discriminadas en la sociedad, no teniendo acceso al poder, la igualdad, etc. Por ello, no nos parece raro que el $70 \%$ de los pobres en el mundo sean mujeres.

La pobreza está muy relacionada con el mercado laboral, una vez que las mujeres encuentran muchas desventajas en tal ámbito. Así, es importante tratar brevemente sobre el tema.

En cuanto al mercado laboral, según Abramo (2006), la tasa de participación de las mujeres pobres en relación a la tasa de participación de los hombres es muchísimo menor, lo que significa que las mujeres más pobres y menos escolarizadas pasan por muchos más problemas para ingresar en el mercado laboral. Aún, señalamos que ellas tienen que conciliar trabajo con responsabilidades domésticas, principalmente la de cuidar de hijas e hijos.

Por lo tanto, es necesario que las tareas domésticas sean redistribuidas, a fin de intentar terminar con la llamada doble jornada de trabajo de las mujeres, que en general, acumulan las tareas profesionales con las ejercidas en el ámbito doméstico que tradicional y culturalmente se mantiene como de su exclusiva responsabilidad.

Según Waring apud Mellor (2007, p. 36), "la invisibilidad de la mujer, del trabajo de la mujer y de las necesidades de la mujer se encuentra en el fondo de los sistemas de toma de decisiones en todo el mundo". No se incluye en el cálculo de la riqueza el trabajo no remunerado de las mujeres y también las necesidades de ellas no son prioridades en los gastos públicos. Además, "los programas de ayuda ignoran a menudo la necesidad de la mujer de cosas tan fundamentales como acceso al agua potable o saneamiento" (DAVIDSON apud MELLOR, 2007, p. 36).

Por lo tanto, podemos constatar que está socialmente introducida la idea de vincular el trabajo doméstico sólo a las mujeres. Por eso, es muy difícil obtener una concienciación para la igualdad, una vez que tal concepción es culturalmente aceptada y reproducida en todos los ámbitos de la sociedad.

En las áreas rurales la pobreza es especialmente aguda, y la disponibilidad de servicios y oportunidades de empleo son menores que en las ciudades. Para las mujeres este hecho aún es más complicado, pues ellas poseen menores posibilidades de acceder a los recursos, factores y servicios productivos tales como el crédito, la propiedad de la tierra, la herencia, la educación, la capacitación, la información, los servicios de extensión, la tecnología y todos los demás recursos, y además están imposibilitadas para participar de forma amplia en los procesos de toma de decisiones.

También en las zonas rurales, en el caso de las mujeres de dicho medio ${ }^{11}$, hay otro factor que dificulta la situación de ellas y que es conciliar familia y empleo. Sin embargo, "no es un tema sobre el que se haya reflexionado o investigado mucho en el ámbito de la sociología rural"'(SAMPEDRO, 2008, p. 85).

Según Sampedro (2008, p. 92):

En el medio rural existen toda una serie de obstáculos estructurales e ideológicos que dificultan el camino hacia la conciliación. A medio plazo, esos obstáculos pueden suponer un problema importante para el desarrollo de las tareas rurales, ya que las mujeres jóvenes seguirán abandonando el medio rural, y con ellas se desvanecerá toda la posibilidad de un futuro viable.

Hay un fuerte impacto del cambio del clima en el objetivo de erradicar la pobreza y el hambre, pues los efectos del cambio climático perjudicarán principalmente a los países en desarrollo y también afectarán a las mujeres de un forma especial, pues 
como ellas ya son pobres y no tienen acceso a varios recursos serán las más perjudicadas.

Es importante decir que acciones de asistencia a los países más pobres como hacen los países más ricos no sirve de nada para la resolución de los problemas de pobreza de estos países. Además, de preservativos y comida debería ser enviado a estos países tecnología, para que desarrollasen sus propios alimentos y la educación; es cierto que solamente la educación tiene la capacidad de cambiar un país, incluso este escenario de pobreza y desigualdad extrema en relación a las mujeres.

La transferencia de tecnología y erradicación de la pobreza están presentes en la Convención Marco de las Naciones para el Cambio Climático, incluso hacen parte y son requisitos de los Mecanismos para el Desarrollo Limpio (MDL), pero esta herramienta no es implementada de manera igual en el mundo. Los datos del Consejo Ejecutivo enseñan que los proyectos de MDL se distribuyen prácticamente entre tres países que hacen parte del BRIC ${ }^{12}$, o sea, Brasil (7\% de los proyectos), China (38\% de los proyectos) e India (27\% de los Proyectos) (BRASIL, MINISTÉRIO DE CIENCIA E TECNOLOGIA, 2010).

Por ello creemos que con o sin mecanismos, con base en la propia Convención Marco, tiene que ser prioritaria la transferencia de tecnología hacia los países más pobres en contra de políticas de asistencia, para que se produzcan en estos países oportunidades de empleo, desarrollo de la educación, disminución de los impactos negativos del cambio climático; y consecuentemente disminución de la pobreza y de las diferencias de género. A partir de esta idea, será estudiado en el apartado siguiente, el acceso a la educación.

El segundo $\mathrm{ODM}^{13}$, es "lograr la enseñanza primaria universal". A través de la educación las personas pueden decidir sobre el estilo de vida que desean llevar. Además, les posibilita manifestarse con seguridad en sus relaciones personales, en la comunidad y en el trabajo. Pero, "hay más de 115 millones de niños en edad de asistir a la escuela primaria que, al no poder acudir a ella, se ven privados de ejercer este derecho humano". Estas niñas y niños, generalmente vienen de hogares pobres, y sus madres en la mayoría de las veces tampoco han recibido educación formal (NACIONES UNIDAS, 2009).

La enseñanza primaria es fundamental para la formación de niñas y niños, pues ofrece toda la base educativa y también abre camino para que se pueda seguir estudiando, y con eso ofrece el acceso a igualdad de oportunidades, generando conocimiento y desarrollo.

Es importante citar que la vulnerabilidad de las mujeres, especialmente de las pobres, aumenta porque la discriminación existe y se reproduce también en el seno del hogar. Muchas veces, si no hay medios para mandar a hijas e hijos a la escuela, los padres prefieren invertir en la educación de los varones, mientras que las niñas se quedan en la casa para compartir las tareas domésticas o en alguna actividad generadora de ingresos (AGUILAR y ARAUJO, 2009).

Se verifica con eso que también se debe dar un cambio cultural, una educación para la igualdad, donde se busque enseñar el cambio en los diferentes grupos sociales, tanto en países en desarrollo como en los desarrollados, pues la desigualdad genera una serie de consecuencias muy serias para toda la sociedad.

La educación sufre muchas limitaciones en los países en desarrollo. Si relacionamos las limitaciones con las consecuencias del cambio climático, verificamos que él "aumenta la carga de trabajo para la producción agrícola y otras actividades de subsistencia como la recolección de agua y leña, lo cual podría presionar a las familias para que saquen a sus hijos e hijas de la escuela". Además, "el extender el tiempo que ellas dedican a realizar esta tarea pone en riesgo su capacidad para asistir a la escuela" (AGUILAR, CASTAÑEDA y CHAO, 2008, p. 30).

Otros efectos del cambio climático que limitan la educación son los desastres naturales y la sequía, que reducen el tiempo disponible de niñas y niños (que se desvía al trabajo doméstico). También los desplazamientos y las migraciones asociadas a este tipo de eventos disminuyen las oportunidades de recibir una educación adecuada

Además, la educación tiene un papel muy importante y directo en promover la capacidad de adaptación a los efectos del cambio climático.

Por lo tanto, se verifica que deben ser realizadas una serie de políticas internacionales para que se puedan disminuir los efectos del cambio climático y así promover la educación. Tenemos conciencia de que no es solo el cambio climático que limita el acceso a la educación; que también hay muchos países pobres con carencia de recursos, que hay sociedades con fuerte influencia de religiones y cuestiones culturales muy fuertes, etc., pero el cambio climático también es un factor para contribuir con la falta de acceso a la educación, y sin educación, principalmente de niñas, no vamos a conseguir la igualdad para que se produzca el cambio social.

El tercer ODM 14 es "promover la igualdad entre los géneros y la autonomía de la mujer”.

El tema de la igualdad ha sido previamente desarrollado, por eso vamos a hacer una breve consideración sobre empoderamiento y así abordar directamente los efectos del cambio climático. 
El empoderamiento debe ser comprendido como un proceso de cambio de las relaciones de poder con la eliminación de las acciones de subordinación y subversión de prácticas instituidas, especialmente en cuanto al ejercicio del poder (MATOS, 2005, p. 80).

El empoderamiento es esencial para que las mujeres tengan voz frente a la lucha feminista, pues así van a poder obtener mayor participación en los procesos de toma de decisión, y participación política, entre otros. En el caso de la lucha contra el cambio climático, es muy importante tratar sobre el empoderamiento de las mujeres y también vincular empoderamiento a la autonomía de las mujeres, porque en varios casos en que las mujeres se ven afectadas por el cambio del clima, muchas veces son víctimas porque no tienen autonomía y eso genera graves consecuencias y es un espejo más de las desigualdades de género.

Es muy importante en este estudio tener en cuenta que:

Los efectos del clima extremo, como inundaciones, deslizamientos de tierra y tormentas son causantes de muerte y lesiones y pueden afectar a mujeres y hombres de diferente manera, dependiendo de la autonomía y los medios a disposición de cada uno para garantizar su propia seguridad. En distintas regiones del mundo se ha podido observar que las restricciones de autonomía y empoderamiento de las mujeres dificultan su acceso a un refugio o a la atención médica en el contexto de ciclones, terremotos e inundaciones (PNUD, 2008, p.49).

La autonomía influye mucho en situaciones de desastres, y por cuestiones culturales, las mujeres son las más perjudicadas, muchas veces son víctimas fatales. Por eso, vamos a mencionar algunos casos de desastres y la diferencia de como se ven afectados mujeres y hombres.

Según Tranter $\left(2008, \mathrm{~s} / \mathrm{p}^{15}\right)$, en Sri Lanka nadar y trepar a los árboles son actividades enseñadas especialmente a los niños. Tal hecho "ayudó más a los hombres que a las mujeres, y les permitió sobrevivir más que a las mujeres cuando les golpeó el tsunami”. Así, se verifica que existe un prejuicio social que imposibilita a niñas y mujeres aprender a nadar, lo que disminuye seriamente sus oportunidades de supervivencia frente a las inundaciones. "A menudo, las mujeres permanecen dentro de sus hogares debido a las prohibiciones sociales en cuanto a dejar la casa".

Además, es muy relevante citar algunos casos concretos, como:

En Aceh, muchas mujeres fueron halladas muertas con bebés sujetados en sus brazos. Algunos relatos personales de sobrevivientes describen a madres empujando a sus niños hacia los techos o copas de los árboles que resistieron el tsunami, pero después fueron arrastradas por el mar. Los largos vestidos que las mujeres son obligadas a usar por las leyes de la sharia de Aceh, hacen muy difícil que puedan moverse con rapidez. No pueden correr tan rápido como los hombres, ni tampoco nadar. Hubo historias de algunas mujeres que estaban dentro de sus casas vestidas de manera informal cuando asaltó la primera ola, que corrieron a ponerse ropas "aceptables" antes de salir a pedir ayuda, y como resultado se ahogaron o apenas pudieron escapar (TRANTER, 2008, $\left.\mathrm{s} / \mathrm{p}^{16}\right)$

Eso es un ejemplo más de las desigualdades de género y de que la cultura influye muchísimo en las relaciones sociales de género. Que las mujeres están sometidas y subordinadas no solamente a los hombres, sino también a las sociedades, a las culturas. Es muy indignante ver que por cuestiones culturales las mujeres, sus hijas e hijos paguen con sus propias vidas. Aquí tenemos una realidad muy dura: efectos gravísimos del cambio climático; y muchas son víctimas además del cambio climático de sus propias culturas.

Es importante que se den las necesarias modificaciones en el hecho de que hasta el momento, en las negociaciones internacionales sobre cambio climático, no se ha verificado la presencia de mujeres en los procesos de toma de decisiones.

Por lo tanto, la igualdad de género influye en varias cuestiones, como, por ejemplo, en las que hemos analizado en este y en otros apartados. También tendrá consecuencias en la salud humana, que será tema de análisis en el apartado siguiente.

El cuarto, el quinto y el sexto $\mathrm{ODM}^{17}$ son "reducir la mortalidad infantil"; "mejorar la salud materna" y "combatir el VIH/SIDA, el paludismo y otras enfermedades".

La Organización Mundial de la Salud (2003, p. 16) en su informe sobre la salud en el mundo del 2003, estimó que "el cambio climático fue responsable en el 2000 de aproximadamente el $2,4 \%$ de los casos de diarrea en todo el mundo y del $6 \%$ de los casos de paludismo en algunos países de ingresos medios". 
Se ha reconocido extensamente que el aumento de los niveles del agua, asociados al cambio del clima conducirán a un aumento de enfermedades relacionadas al agua. Otras consecuencias probables del cambio del clima en la salud incluyen índices más altos de la desnutrición debido a la falta de alimentos, a los aumentos de mortalidad y morbosidad relacionados con el calor, y a las enfermedades respiratorias crecientes donde la contaminación atmosférica se empeora. Los niños por debajo de cinco años y los ancianos son las víctimas principales de enfermedades relacionadas con el saneamiento. La discriminación de género en la asignación de recursos, incluyendo los referentes a la nutrición y a las medicinas, puede poner a las chicas en mayor riesgo que los chicos. Más investigación en los impactos de género, específicos a la salud y el cambio del clima ayudaría a eliminar el grado en que esto está ocurriendo en cada caso, y permitiría un mayor compromiso (BRODY, DEMETRIADES y ESPLEN, 2008).

En muchos países pobres las personas casi no tienen qué comer, lo que lleva a la desnutrición, además del agua no tratada y consecuentemente hacen que chicos y chicas sean más vulnerables a las enfermedades. La relación desnutrición y enfermedad son interdependientes, y con el cambio climático las noticias de que los problemas en relación a los alimentos aumentarán debido al clima y suelo, solamente hacen que sean las medidas tecnológicas juntamente con la disminución de las emisiones tomadas democráticamente.

Actualmente, se verifica en el mundo entero un aumento de la periodicidad de muchas enfermedades infecciosas, incluidas algunas de reciente aparición, como VIH/SIDA, hantavirosis, hepatitis $\mathrm{C}$, etc. Eso manifiesta el impacto combinado de los rápidos cambios demográficos, ambientales, sociales, tecnológicos y de otro tipo sobre nuestros modos de vida (PATZ, 2003).

El cambio climático afecta a la salud de las mujeres de diferentes formas. Primero, considerando el típico rol de las mujeres como responsables de la salud de la familia se incrementa su carga de trabajo y la probabilidad de contagiarse de enfermedades infecciosas, como el paludismo que genera anemia y es responsable de una cuarta parte de la mortalidad materna. Las mujeres embarazadas son particularmente susceptibles a las enfermedades asociadas al agua y otros vectores (PATZ, 2003).

El aumento de las temperaturas (olas de calor), los altos índices de la mortalidad de madres/mujeres/esposas durante los desastres resultan con un aumento en los números de huérfanos y de tasas de la mortalidad; uniones tempranas para las chicas jóvenes (nuevas esposas) lo que hace que ellas dejen la escuela; el tráfico y la prostitución aumentan la exposición a VIH/SIDA (OXFAM, 2008). Es importante decir que el SIDA genera desempleo y eso contribuye aún más a la situación de pobreza de las mujeres.

Según la Organización Mundial de la Salud (2003), aunque sean adoptadas acciones para mitigar el cambio climático, será necesaria la adaptación orientada a proteger la salud pública, pues la adaptación al cambio climático requerirá más que recursos económicos, tecnología e infraestructuras de salud pública; también será necesario instruir, concienciar y crear marcos jurídicos, instituciones y un entorno que permita adoptar decisiones sostenibles, duraderas y bien fundamentadas.

Así, constatamos que desigualdad de género, salud humana y cambio climático se relacionan y también generan víctimas fatales. Por eso, son necesarias una serie de políticas a fin de disminuir las graves consecuencias en la salud de las personas, porque debemos preocuparnos en tener un medio ambiente sostenible, que será tema del próximo apartado.

El séptimo ODM" es "garantizar la sostenibilidad del medio ambiente".

Las medidas de adaptación para el cambio climático, necesitan de mucho trabajo. También, son escasos la participación de las mujeres y el número de representantes en el ámbito de toma de decisiones sobre el cambio climático, incluyendo a todos los ámbitos, o sea, local, nacional, regional e internacional. Por lo tanto, la mayor parte de las políticas sobre cambio climático no reflejan las ideas, necesidades y prioridades de las mujeres (AGUILAR; ARAUJO; QUESADA-AGUILAR, 2009)

Para obtener la sostenibilidad del medio ambiente es primordial que los recursos naturales sean utilizados de manera inteligente y que sean protegidos los ecosistemas complejos de los cuales depende nuestra supervivencia. "Debe tenerse en cuenta que, la sostenibilidad no podrá lograrse con los modelos actuales de consumo y uso de recursos" (NACIONES UNIDAS, $2009^{19}$ ).

Una vez más vamos a opinar que hay una gran preocupación con la pobreza y que las mujeres son las principales integrantes de esta clase social y que están muy afectadas por diversos factores. Así, en los países pobres no hay respeto por el medio ambiente, pues son explotados por los países ricos, por eso hay una gran necesidad de que sea promovida la sostenibilidad del medio ambiente por los países ricos a fin de frenar los efectos del cambio del clima.

Por ello y para finalizar este apartado, es importante que reflexionemos, de acuerdo con Velayos 
(2007), en torno a la igualdad y la sostenibilidad, pues la lucha por la igualdad pide, conforme el feminismo ecológico 'recuperar la otra voz', la voz de todas aquellas mujeres que no han ejercido el dominio objetivista de la naturaleza y cuyo reconocimiento puede ser crucial para iniciar un cambio de actitud". Velayos (2007, p. 98) menciona la Declaración Mundial de Río de Janeiro, que determina en su principio 20 que: "las mujeres desempeñan un papel fundamental en la ordenación del medio ambiente y en el desarrollo". Por lo tanto, podremos afirmar que es indispensable la total participación de las mujeres para alcanzar un desarrollo sostenible.

\section{Conclusiones}

En este estudio hemos podido constatar que el cambio climático tiene y va a seguir teniendo efectos muy graves, como un aumento en el nivel del mar, cambios en los patrones de la precipitación pluvial, mayor riesgo de sequías e inundaciones, amenazas a la biodiversidad y potenciales desafíos para la salud pública.

Por ello, tales efectos afectan mayoritariamente a los débiles de las sociedades, que son las mujeres, las niñas y los niños, lo que hace más difícil que mantengan su derecho a la salud, a la alimentación, al agua, a la educación, etc. Así, podremos afirmar que el cambio climático además de ser un asunto de medio ambiente también es un tema de derechos humanos.

Por lo tanto, al tratar sobre el cambio climático debemos tener en cuenta el contexto social, económico y político que lo determina. Así, debemos considerar los temas relacionados con la pobreza y el desarrollo, pues para que se pueda superar la pobreza y promover el desarrollo se tiene que entender que ambos se distribuyen de manera desigual, afectando a mujeres y hombres de manera diferenciada.

La cultura en determinadas sociedades perjudica aún más a las mujeres porque las hace víctimas fatales de los efectos del clima ya que en situaciones de desastres ambientales, por ejemplo, las mujeres mueren en mayor número puesto que tienen el papel de cuidadoras y además porque no están preparadas para defenderse frente a esas situaciones calamitosas. También las mujeres tienen el acceso a la educación más limitado, lo que no fomenta la igualdad de género.

Además, las mujeres ven afectada su salud por consecuencia del cambio climático, pues hay posibilidad de propagación de enfermedades infecciosas como SIDA y paludismo. El SIDA hace con que haya una gran limitación en la vida de muchas mujeres, llevando muchas a la muerte o contribuye aún más para la pobreza porque genera el desempleo. El paludismo afecta y puede a llevar a la muerte a las mujeres embarazadas, pues genera anemia. Eso ocurre mucho en África, donde hay carencia de recursos.

Las mujeres deben ser tenidas en cuenta para que se pueda lograr éxito en la implementación de programas y en la mitigación de los efectos del cambio del clima. Además, las mujeres utilizan su ambiente de manera diferente que los hombres, pues en la mayoría de las veces están más sensibilizadas con causas ambientales y también más conscientes; también por el hecho de la forma como se ven afectadas por el cambio climático; por todo eso, se nota que estos ejemplos son factores importantes en un programa exitoso de cambio climático.

Imprescindible es introducir el enfoque social, económico, político y cultural en el análisis del origen y de los efectos del cambio climático, especialmente, incorporar el examen de las relaciones de género. Incluso es preciso desarrollar metodologías con enfoque de género en los diferentes contextos asociados con el cambio del clima e insertarlas en políticas, programas y proyectos.

También es preciso que se dé una cooperación en todos los ámbitos (local, regional, nacional e internacional), para promover estrategias de adaptación y también de educación. Es obligado introducir en el ámbito nacional e internacional medidas legales relacionadas a la igualdad de género con el fin de lograr la igualdad real y efectiva.

Imprescindible es destacar, que las políticas públicas tienen un papel muy importante en la redistribución de los recursos en la sociedad para que se alcance de igual forma a mujeres y hombres. Además, acrecentar el enfoque de la igualdad de género en su elaboración e implementación posibilita profundizar su impacto en la corrección de las desigualdades sociales que agravan las consecuencias del cambio del clima. Incluso deben ser creados medios y oportunidades equitativas para la educación e información para el cambio del clima.

Por lo tanto, es esencial que se promueva la participación de las mujeres en el debate y en la toma de decisiones relacionadas con el cambio climático, y también que sea fomentada una representación igual entre mujeres y hombres para que se pueda garantizar que el enfoque de género sea perceptible y que las mujeres logren voz activa y se dé el empoderamiento que les corresponde.

1 Disponível em

http://www.revistafuturos.info/futuros 16/cambioclima genero.htm. Acesso em 14 de março de /2011. s/p.

${ }_{2}$ Es importante informar que la cuestión 
cultural influye mucho en el comportamiento de las mujeres y eso hace con que en situaciones de desastre, por ejemplo, ellas sean víctimas en número mucho mayor, pues además del ejemplo de no ser enseñadas a nadar, muchas veces son ellas que se sienten responsables por intentar sacar todas las personas (menores y ancianos) de la viviendas, y eso se atribuye al papel de cuidadoras que tienen las mujeres. En algunas sociedades, en las islámicas, por ejemplo, las mujeres en una situación de desastre se preocupan en estar con el cuerpo totalmente cubierto para poder salir de casa y eso hace que tarden más en intentar salvarse.

3 La capa de ozono se encuentra en la estratosfera, siendo un contaminante importante desde que se quede en esta capa, pues en ciudades como la Ciudad de México hay ozono en la troposfera provocando el "smog". El "smog" es muy perjudicial a la salud humana al concentrar los contaminantes en la troposfera, provocando enfermedades como las respiratorias (HARDY, 2005, p. 45).

$4 \quad$ Veáse NICHOLSON, Linda. Interpreting gender. En: Signs, v. 20, n.1, p. 79-105, Chicago: University of Chicago Press, 2004.

5 Disponível em <

http://www.undp.org.gt/odm/homex.htm>.Acesso em 21 de março de 2011. s/p.

6 Disponible

$<$ http://www.ucm.es/info/mageeq/glosario.htm>.

Acceso en 20 de agosto de 2008.

7 Sobre la Ética del cuidado, véase GILLIGAN,

Carol. In a different voice: psychological theory and women's development. Massachusetts: Harvard University Press, 1993.

$8 \quad$ Disponivel

em

$<$ http://www.jornada.unam.mx/2007/11/27/index.php?s ection $=$ sociedad\&article $=044 \mathrm{n} 1 \mathrm{soc}>$.Acesso em $15 \mathrm{de}$ março de /2011. s/p.

9 Disponível

em

$<$ http://www.gendercc.net/fileadmin/inhalte/Dokument e/Tools/Toolkit_Genero_en_la_pol\%C3\%ADtica_clim \%C3\%A1tica.pdf> Acesso em 15 de março de 2011, p.13.

10

Disponível

em

$<$

http://www.undp.org.gt/odm/homex.htm>.Acesso em 21 de março de 2011. s/p.

11 En cuanto a las mujeres rurales y el ámbito doméstico, es muy importante hacer una breve citación sobre la identidad, que de acuerdo con MAYA FRADES, Valentina (Ed.): Señas de identidad de la mujer rural, en Mujeres rurales - Estudios multidisciplinares de género, Ediciones Universidad de Salamanca, Salamanca, 2008, pág. 30: “La mujer agraria se concibe y es percibida como mujer rural, identidad que le viene dada por su actividad, pero ello no quiere decir que al hablar de ella nos encontremos con un único perfil. El trabajo agrario de las mujeres de las generaciones mayores ha sido socialmente opaco, muchas veces, por el carácter familiar de la explotación, donde desde fuera sobresalía la función de ama de casa, incluyendo todos los roles que la mujer ejerce en el ámbito privado, desde los cuidados familiares hasta su trabajo en la explotación familiar que ha sido considerado como un complemento o ayuda (trabajo invisible)".

12 Expresión creada por el economista JIM O'NEILL en el informe "Building Better Global Economic Brics", los cuatro principales países emergentes del mundo, los cuales son considerados Brasil, Rusia, India y China.

13 Disponível em < http://www.undp.org.gt/odm/homex.htm $>$.Acesso em 21 de março de 2011. s/p.

14 Disponível em <

http://www.undp.org.gt/odm/homex.htm $>$.Acesso em 21 de março de 2011. s/p.

15 Disponivel em $<$ http://www.ecoportal.net/Temas_Especiales/Cambio_ Climatico/Mujeres_y_cambio_climatico $>$. Acesso em 15 de março de 2011. s/p.

16 Disponivel em $<$ http://www.miradaglobal.com/index.php?option=com _content\&view=article\&id=837:mujer-y-cambio-

climatico\&catid=32: ecologia\&Itemid $=36 \&$ lang $=e s>$. Acesso em 15 de março de 2011. s/p.

17 Disponível em

http://www.undp.org.gt/odm/homex.htm>.Acesso em 21 de março de 2011. s/p.

18 Disponível em < http://www.undp.org.gt/odm/homex.htm $>$.Acesso em 21 de março de 2011. s/p.

19 Disponível em < http://elzocolaprimeraalaizquierda.org/revista/sociedad -revista/derechos-civiles/objetivo-del-milenio-7garantizar-la-sostenibilidad-del-medio-ambiente>.

\section{Referências}

ABRAMO, Laís. Desigualdades de gênero e raça no mercado de trabalho brasileiro. Ciência e Cultura SBPC, vol. 58, no 4, p. 40-41, 2006.

AGUILAR, Lorena; ARAUJO, Ariana; QUESADAAGUILAR, Andréa. Gender and climate change. IUCN. Disponible en: $<$ http://generoyambiente.org/admin/admin_biblioteca/ documentos/Factsheet $\% 20$ ClimateChange.pdf,Acceso en 15 de julio de 2009. 
AGUILAR, Lorena; CASTAÑEDA, Itzá; CHAO, Verania. PROGRAMA DE LAS NACIONES UNIDAS PARA EL DESARROLO (PNUD). Guía Recursos de género para el cambio climático. México, 2008.

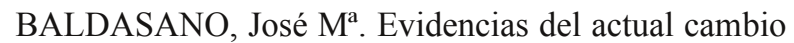
climático. Cambio climático y sus consecuencias. In: PRESIDÈNCIA DE LA GENERALITAT VALENCIANA, Valencia: Generalitat Valencia, 2007. p. 43-56.

BRASIL, MINISTÉRIO DA CIÊNCIA E TECNOLOGIA. Status atual dos projetos de MDL no Brasil e no mundo. Disponible en:

$<$ http://www.mct.gov.br/index.php/content/view/30317 .html>.

Acceso en: 29 de septiembre de 2010.

BRODY, Alyson; DEMETRIADES, Justina; ESPLEN, Emily. BRIDGE - INSTITUTE OF DEVELOPMENT STUDIES, Gender and climate change: mapping the linkages - A scoping study on knowledge and gaps, 2008. Disponible en: $<$ http://www.bridge.ids.ac.uk/>. Acceso en 10 de mayo de 2008.

CAMPOS PEREGRINA, $\mathrm{M}^{\mathrm{a}}$ del Carmen; PEREZ VARGAS, $\mathrm{M}^{\mathrm{a}}$ José. Breve Guía sobre cambio climático y género y cooperación al desarrollo y género. Fundación Gondwana para el desarrollo sostenible. 2007.

MELLOR, Mary. "Mujer, naturaleza y construcción social del hombre económico". En: BARRIOS, Olga; FIGUERUELO, Ángela; LÓPEZ, Teresa; VELAYOS, Carmen (Eds.). Feminismo ecológico - Estudios multidisciplinares de género. Salamanca: Ediciones Universidad de Salamanca, 2007.

DOMINGUES SALINAS, Elena. Coordinado por FUNDACIÓN PARA EL DESARROLLO. Guía básica sobre cambio climático y cooperación para el desarrollo. Madrid: Genia Producción Gráfica. DUDDY, Janice. Es el cambio climático un as

unto de género? Disponible en: $<$ http://www.nodo50.org/mujeresred/spip.php?article5 9>. Acceso en 14 de septiembre de 2010.

GONZÁlEZ GARCÍA, Marta I. Habitando los espacios naturales en cuerpos sexuados: género y responsabilidad medioambiental. ARBOR: ciencia, pensamiento y cultura, Cualificar el espacio: transiciones ambientales para el nuevo milenio. CLXXXIV. 729. Consejo Superior de Investigaciones Científicas, enero-febrero. p.115. Madrid, 2008.

GORDON, Bruce; MACKAY, Richard; REHFUESS, Eva. Inheriting the World: The Atlas of Children's Health and the Environment. Hong Kong: Fenix Offset, 2004.

HARDY, John T. Climate change: Causes, effects, and solutions. Wiley: West Sussex, 2005.

LAGARDE, Marcela. Género y feminismo: desarrollo humano y democracia. Madrid: Horas y horas, 1996.

MATOS, Maria Izilda. Terceiro setor e gênero: trajetórias e perspectivas. São Paulo: Cultura Acadêmica: Instituto Presbiteriano Mackenzie, 2005.

MURILLO, Soledad. El mito de la vida privada. De la entrega al tiempo propio. Madrid: Siglo XXI, 1996.

NACIONES UNIDAS,. Disponible en: $<$ http://www.un.org/spanish/millenniumgoals/educatio n.shtml $>$. Acceso en 10 de junio de 2009.

NARAYAND, Deepa. Voices of the poor - Can anyone hear us? Washington, DC: The World Bank Oxford University Press, 2000.

NICHOLSON, Linda. Interpreting gender. Signs, vol. 20, no 1, p. 79-105, 2004.

ORGANIZACIÓN DE LAS NACIONES UNIDAS PARA LA AGRICULTURA Y LA ALIMENTACIÓN. Disponible en: $<$ http://www.fao.org/Gender/static/Method/2statds1.ht $\mathrm{m}>$. Acceso en 15 de junio de 2009.

ORGANIZACIÓN MUNDIAL DE LA SALUD OMS. Cambio climático y salud humana riesgos y respuestas: resumen. Ginebra: OMS, 2003.

OXFAM. The tsunami's impact on women. 2005. Disponible en: $<$ http://www.oxfam.org/en/files/bn050326_tsunami _women/download.> Acceso en 16 de julio de 2009.

ENCISO, Angelica. "El cambio climático ahonda desigualdades sociales y revertirá el desarrollo: PNUD". Disponible en: $<$ http://www.jornada.unam.mx/2007/11/27/index.php? 
section $=$ sociedad\&article $=044 \mathrm{n} 1$ soc $>$ Acceso en $10 \mathrm{de}$ mayo de 2009.

RUBIO DE URQUìA, Francisco Javier. El cambio climático más allá del Protocolo de Kyoto Elementos para el debate. Madrid: Ministerio del Medio Ambiente, 2006.

SAMPEDRO GALLEGO, Rosário. Conciliacón de la vida familiar y laboral en el medio rural: género, trabajo invisible e "idilio rural. En: MAYA FRADES, Valentina (Ed.). Mujeres rurales - Estudios multidisciplinares de género. Salamanca: Ediciones Universidad de Salamanca, 2008.

SCOTT, Joan. Gênero: uma categoria útil de análise histórica. En: Educação e Realidade. vol. 20, nº 2. p. 71-99, 1995.

Tercer Informe de Evaluación. Cambio Climático 2001. Impactos, Adaptación y Vulnerabilidad (IPCC), 2001.

TRANTER, Kellie. Mujeres y cambio climático. En: Revista Sin Permiso. 10 de marzo de 2008. Disponible en:

nnnnnnnn $<$ http://www.sinpermiso.info/textos/index.ph p?id=1720 $>$. Acceso en 10 de marzo de 2009.

UNITED NATIONS DEVELOPMENT PROGRAMME (UNDP). Informe de Desarrollo Humano 2007-2008, 2008.

VELAYOS, Carmen. Ética y cambio climático. Bilbao: Desiée De Browwer, 2008.

VELAYOS CASTELO, Carmen. Sostener la vida: la ética ecofeminista. En: BARRIOS, Olga; FIGUERUELO, Ángela; LÓPEZ, TERESA; VELAYOS, Carmen (Eds.). Feminismo ecológico Estudios multidisciplinares de género. Salamanca: Ediciones Universidad de Salamanca, 2007.

MELLOR, Mary. "Mujer, naturaleza y construcción social del hombre económico". En: BARRIOS, Olga; FIGUERUELO, Ángela; LÓPEZ, TERESA; VELAYOS, Carmen (Eds.). Feminismo ecológico Estudios multidisciplinares de género. Salamanca: Ediciones Universidad de Salamanca, 2007. 